\title{
Hybrid BPSK-modified MPPM: A Scheme for Enhancing Optical MPPM in Optical Fiber Communications
}

\author{
H. Selmy, H. M. H. Shalaby and H. S. Khallaf \\ Department of Electronics and Communications \\ Engineering \\ Egypt-Japan University of Science and \\ Technology (EJUST) \\ Alexandria 21934, Egypt \\ Email: hossamselmy@yahoo.com
}

\author{
Z. Kawasaki \\ Department of Electronics and Communications \\ Engineering \\ Graduate School of Engineering, Osaka University \\ Suita, Osaka 565-0871, Japan \\ Email: zen@comm.eng.osaka-u.ac.jp
}

\begin{abstract}
A hybrid binary phase shift keying-modified multipulse pulse-position modulation (hybrid BPSK-modified MPPM) scheme is proposed as a new modulation technique to improve both the symbol-error rate performance and bandwidth utilization efficiency of conventional optical multi-pulse pulse position modulation (MPPM) scheme in optical fiber communication systems. Whereas in conventional MPPM scheme, unmodulated pulses are transmitted in every signal block, BPSK modulated pulses is transmitted in the proposed hybrid scheme. That is, the information is encoded in both the positions and phases of the transmitted pulses. The transmission characteristics, transmitter and receiver structures, bandwidth utilization, and optimum decoding for the proposed scheme are presented in this paper. Several performance measures are derived and compared to those of conventional MPPM schemes, adopting both coherent and direct detection receivers, under the same data transmission rates. Our results reveal that, at same average power levels, the proposed hybrid BPSK-modified MPPM scheme achieves much lower levels of symbol-error rates compared to those of ordinary MPPM schemes. Furthermore, in terms of bandwidth-utilization efficiency, the proposed hybrid modulation scheme achieves much higher efficiencies than that of ordinary MPPM schemes.
\end{abstract}

Keywords—Optical modulation; BPSK; MPPM.

\section{INTRODUCTION}

Pulse-position modulation technique represents an important modulation category for both optical fiber and free space optical communications systems. One important format of this category is multi-pulse pulse-position modulation (MPPM) scheme, which has been proposed to enhance the bandwidth utilization efficiency of ordinary single-pulse pulseposition modulation (PPM) scheme [1], [2]. Both PPM and MPPM have been used extensively with intensity modulation/direct detection (IM/DD) systems. However, for better receiver sensitivity, coherent detection has been recently proposed (including both homodyne and heterodyne detections) to demodulate such schemes [3], [4]. For shot noise limited systems, the sensitivity of heterodyne detection receivers is better by $3 \mathrm{~dB}$ than that of direct detection receivers. In order to further enhance the performance of pulseposition modulation techniques, hybrid modulations can be used. One direction of such hybrid modulations is to combine both phase and pulse-position modulation schemes in order to exploit the advantages of both of them. The main advantage of using phase modulation is to increase bandwidth utilization efficiency of the hybrid scheme. By combing phase modulation with pulse-position modulation, the average transmitted power will be reduced further, leading to both high bandwidth utilization efficiency and reasonable receiver sensitivity. The idea of hybrid phase and pulse-position modulations for optical communications has been recently proposed in many literatures. Both classical and quantum receivers for hybrid BPSK-2PPM scheme have been investigated and their performances have been compared to each other [5]. Another hybrid modulation, which combines both PPM and binary phase shift keying subcarrier intensity modulation (BPSKSIM), has been proposed in [6]. This scheme offers BER performance that is superior to that of both BPSK-SIM and PPM at the same average transmitted power and bit rate. Recently, an experimental work has been carried out to demonstrate a record sensitivity in an optically pre-amplified receiver by combining both polarization-division-multiplexed quadrature phase-shift keying (PDM-QPSK) and 16-PPM [7]. Pilot-assisted digital coherent detection has been used. The results indicate a $3 \mathrm{~dB}$ theoretical sensitivity advantages over both PDM-QPSK and BPSK in an optically pre-amplified receiver.

In this paper, we aim at enhancing the performance of ordinary multi-pulse pulse-position modulation (MPPM) scheme by combining it with binary phase shift keying (BPSK) format. Specifically, we propose a modified MPPM technique and modulate its signal pulses using ordinary BPSK format. We call the proposed modulation scheme hybrid binary phase shift keying-modified multi-pulse pulse-position modulation (hybrid BPSK-modified MPPM). In this scheme, the number of transmitted bits is increased (compared to traditional MPPM) by encoding extra bits using the BPSK format. Clearly, the number of these excess bits is equal to the 


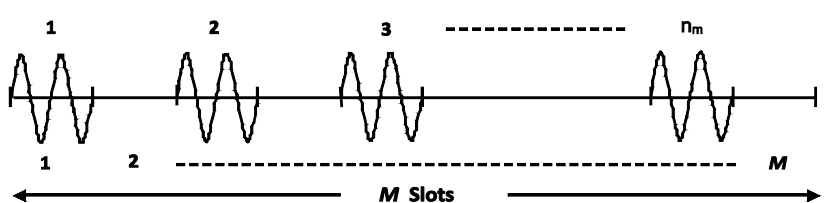

Fig. 1. Frame structure of coherent detection n-pulse MPPM

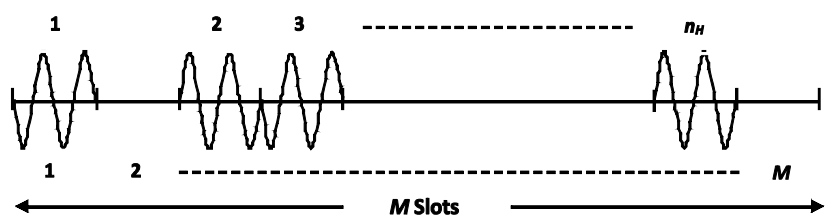

Fig. 2. Frame structure of hybrid n-BPSK-modified MPPM scheme.

number of transmitted optical pulses in the hybrid frame. Also, as the demodulation of BPSK pulses requires the implementation of coherent detection (either homodyne or heterodyne), we propose to use coherent detection for demodulating both BPSK and MPPM symbols in the hybrid frame.

The rest of the paper is organized as follows. In Section II, we develop a general system model for the hybrid BPSK modified MPPM scheme and study its transmission characteristics. Also, the differences between the proposed hybrid scheme and the ordinary scheme are stated. In Section III, we present the transmitter and receiver structures for the hybrid scheme. Also, we state its maximum-likelihood decoding (optimal decoding) in optically pre-amplified channels with amplifier noise limited case. In Section IV, we derive performance measures in terms of the achieved symbolerror rates under both average and peak transmission power constraints. Next, the performance comparisons and numerical results are presented in Section V. Finally, the conclusion is given in Section VI.

\section{Proposed System Model AND CHARACTERISTICS}

Here, we propose a Hybrid BPSK-modified MPPM modulation scheme in which binary phase shift keying (BPSK) is combined with a modified version of multi-pulse pulse position modulation. Precisely, for frames of size $M$ slots, instead of simply transmitting $n_{M}$ unmodulated optical pulses in the ordinary MPPM frame; BPSK is used to modulate $n_{H}$ optical pulses in the hybrid frame. The frame structure of both the proposed hybrid $n$-BPSK-modified MPPM scheme and the coherent detection $n$-pulse MPPM scheme are explained in Figs. 1 and 2, respectively. The term modified MPPM comes from the ability to increase number of transmitted optical pulses per hybrid frame to values more than $M / 2$. This is in contrast to ordinary MPPM in which the maximum number of transmitted optical pulses per frame cannot increase above $M$ / 2 .

Generally, the goal of our proposal of this hybrid technique is to enhance the performance of ordinary MPPM scheme in optical communications. One of the main enhancements of this

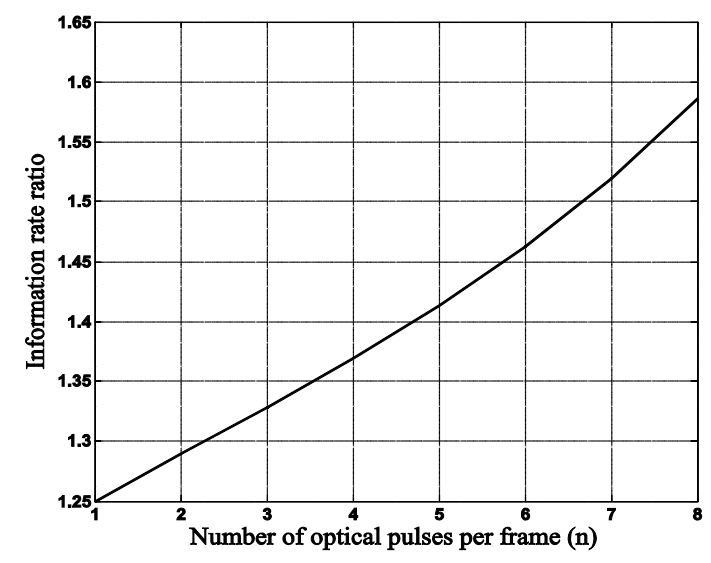

Fig. 3. Information rate ratio for $M=16$.

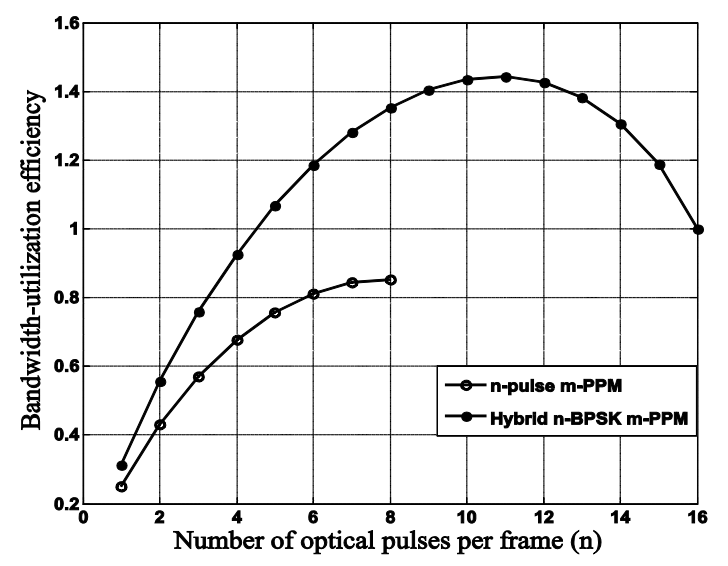

Fig. 4. Bandwidth utilization efficiency.

hybrid scheme is the significant increase in bandwidth utilization efficiency. Clearly, for ordinary MPPM, the number of transmitted bits per frame is $\log _{2}\left(\begin{array}{c}M \\ n_{M}\end{array}\right)$ bits, whereas for the proposed hybrid $n$-BPSK-modified MPPM scheme, the number of transmitted bits per frame is $n_{H}+$ $\log _{2}\left(\begin{array}{c}M \\ n_{H}\end{array}\right)$. Therefore, at the same values of $n_{M}$ and $n_{H}$, i.e., $n_{M}=n_{H}=n$, the information rate ratio for the two schemes is given by

$$
\text { Information rate ratio }=\frac{n+\log _{2}\left(\begin{array}{c}
M \\
n
\end{array}\right)}{\log _{2}\left(\begin{array}{c}
M \\
n
\end{array}\right)}
$$

In Fig. 3, we plot the information rate ratio for the case of $M=16$. The figure indicates that the information rate ratio increases by increasing the number of optical pulses per frame, allowing the transmission of more bits per frame. In other words, the proposed scheme could offer much higher transmission rate than that of the ordinary MPPM scheme with the same number of optical pulses per frame. Also, as stated before, the bandwidth-utilization efficiency is increased significantly using the proposed scheme. Clearly, the bandwidth utilization efficiency for ordinary MPPM scheme is 


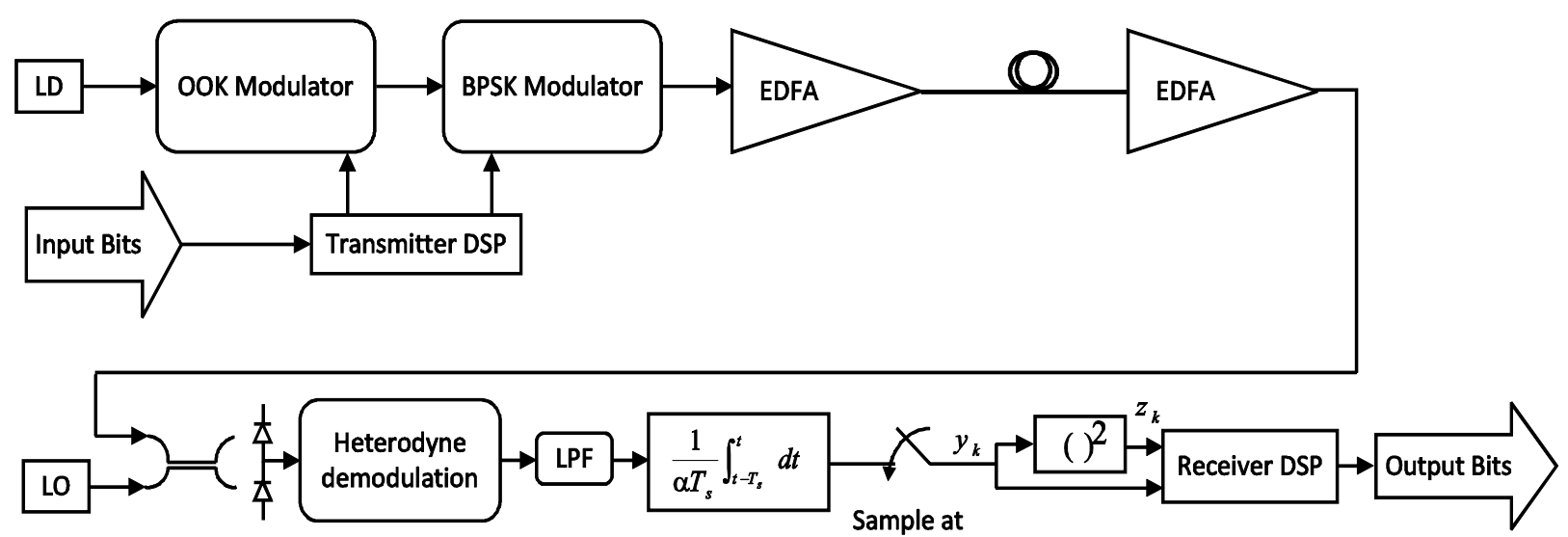

Fig. 5. Transmitter and receiver structures for hybrid BPSK modified MPPM scheme. The parameter $\alpha=2 R A L$ for a homodyne receiver or $\alpha=R A L$ for a heterodyne receiver.

given by

$$
U_{M}=\frac{\log _{2}\left(\begin{array}{c}
M \\
n_{M}
\end{array}\right)}{M}
$$

Whereas for the hybrid BPSK-modified MPPM scheme, it is given by

$$
U_{H}=\frac{n_{H}+\log _{2}\left(\begin{array}{c}
M \\
n_{H}
\end{array}\right)}{M}
$$

The bandwidth-utilization efficiencies for both schemes are compared at a fixed frame size of 16 slots. The comparison is carried out at different values of $n_{H}$ and $n_{M}$, as shown in Fig. 4. Obviously, the figure indicates that the maximum achievable efficiency for the $n$-pulse 16-PPM scheme is about $85 \%$ and it occurs at $n_{M}=8$ pulses, whereas for the hybrid $n$-BPSK 16PPM scheme, the bandwidth utilization efficiency could approach levels higher than $100 \%$. Specifically, for hybrid $n$ BPSK 16-PPM scheme, this efficiency has a maximum of $144.33 \%$ at $n_{H}=11$ pulses. After reaching this maximum value, the efficiency decreases again by increasing the value of $n_{H}$ until approaching a value of $100 \%$ at $n_{H}=16$. This latter case represents the ordinary BPSK transmission. Clearly, from these results, the proposed modulation scheme is strongly intended to be used for applications that require high bandwidth utilization.

\section{TRANSMITTER AND RECEIVER STRUCTURES AND DECODING ALGORITHMS}

The transmitter and the receiver structures for hybrid BPSKmodified MPPM are shown in Fig. 5. At the transmitter side, the transmitted data bits are first fed to a digital signal processing (DSP) device, which divides them into several blocks. Each block contains $\log _{2}\left(\begin{array}{c}M \\ n_{H}\end{array}\right)+n_{H}$ bits. The first $\log _{2}\left(\begin{array}{c}M \\ n_{H}\end{array}\right)$ bits determine the transmitted MPPM symbol, i.e., the positions of the $n_{H}$ signal pulses within the MPPM frame, while, the remaining $n_{H}$ bits are used to modulate these signal pulses with ordinary BPSK modulation. That is, the first $\log _{2}\left(\begin{array}{c}M \\ n_{H}\end{array}\right)$ bits of the data block are encoded in the hybrid frame by intensity modulating a continuous wave $(\mathrm{CW})$ laser source, generating $n_{H}$ optical pulses. The remaining $n_{H}$ bits are encoded in the hybrid frame by phase modulating the $n_{H}$ optical pulses in the hybrid frame. After that, the optical signal is transmitted through an optical fiber and periodically amplified in the fiber span till reaching the receiver side.

At the receiver side, coherent detection, for both BPSK symbols and MPPM frames, is adopted to increase the receiver sensitivity. In addition, the coherent receiver implements balanced optical detectors in order to minimize the noise generated from local oscillator (LO). Generally, for coherent receivers limited by optical amplifier noise, both heterodyne and homodyne detection receivers achieve same performance [8]. The photodetector output of the homodyne receiver (or the IF demodulator output of the heterodyne receiver) is then fed to a slot integrator. The slot integrator output is then squared and both the integrator output and its square value are passed to a DSP to decode the received hybrid frame. The DSP selects the $n_{H}$ pulses with the maximum square values and decode them as the signaling pulses in the received frame. This is the same as normal decoding of MPPM schemes [9]. After decoding the first $\log _{2}\left(\begin{array}{c}M \\ n_{H}\end{array}\right)$ bits of the transmitted data block, the DSP proceeds to decode the remaining $n_{H}$ bits by comparing the slot integrator values for the predetermined $n_{H}$ signal slots with zero. Obviously, the slot is decoded as one if its integrator output is larger than zero; otherwise, it is decoded as zero.

\section{Decoding Algorithm}

1) Perform coherent detection for received hybrid frame by homodyne or heterodyne demodulation.

2) Get received signal amplitude in each slot. 
3) Determine the energy received in each slot by integrating the received signal amplitudes and squaring the integrator output samples.

4) Determine $n_{H}$ signal slots of received hybrid frame by selecting the $n_{H}$ slots with the highest energies.

5) Decode the BPSK signals of these slots by comparing their integrated samples to zero.

\section{PERFORMANCE EVAlUATION}

In this section we aim at evaluating the symbol-error rate (SER) for the proposed hybrid BPSK-modified MPPM. In our evaluation we consider the optical fiber as the transmission medium for the hybrid scheme. Also, we assume that the optical fiber span contains many optical amplifiers so that the system is limited by the amplified spontaneous emission (ASE) noise generated from these amplifiers [8]. Generally, the extension of the carried analysis for both free space optical communications and deep space optical communications is straightforward and can be achieved by considering appropriate optical propagation models of these channels. Here, the input data stream is divided into continuous blocks, each block contains $\log _{2}\left(\begin{array}{c}M \\ n_{H}\end{array}\right)+n_{H}$ bits. Each data block is encoded to form one hybrid frame. Thus, the SER for the hybrid BPSK modified MPPM scheme is the result of two independent events and is given by

$$
\mathrm{SER}_{\mathrm{H}}=1-\left(1-\mathrm{SER}_{\mathrm{MPPM}}\right) \times\left(1-\mathrm{BER}_{\mathrm{BPSK}}\right)^{n_{H}}
$$

Where $B \mathrm{BR}_{\mathrm{BPSK}}$ and $\mathrm{SER}_{\mathrm{MPPM}}$ are the bit error rate of ordinary BPSK and the symbol error rate for ordinary MPPM, respectively. The first parentheses in (4) accounts for the event that the MPPM symbol is correctly decoded and its pulses' positions are correctly determined. The second parentheses of the equation accounts for the event that all BPSK symbols within the hybrid frame are correctly decoded. Clearly, the hybrid frame is considered to be correctly decoded only when both pulses' positions and BPSK symbols are correctly identified. In the analysis of pre-amplifiered coherent receivers there are many types of noise sources. The main three noise sources are the amplified spontaneous emission noise (ASE), the local oscillator noise, and the detector noise, which includes both the shot and thermal noises. Here, we use a dual-photodiode balanced receiver to reduce the LO noise and increase the signal power. Also, we assume that the detector shot noise variance is much larger than that of the thermal noise so that the detector thermal noise can be neglected.

The electric field for both the received optical field and the LO field are given by

$$
\begin{aligned}
E_{r}(t) & =\left[A_{s}(t) e^{j \phi_{S}(t)}+n_{x}(t)\right] e^{j \omega_{c} t} \hat{x}+n_{y}(t) e^{j \omega_{c} t} \hat{y} \\
E_{L O}(t) & =\left[A_{L}+n_{L}(t)\right] e^{j \omega_{L O} t} \hat{x},
\end{aligned}
$$

where, $A_{s}(t), \phi_{s}(t)$, and $\omega_{c}$ are the amplitude, phase, and frequency of the received signal, respectively, $\hat{x}$ is the polarization of the signal, $\hat{y}$ is the polarization orthogonal to $\hat{x}$, $n_{x}(t)$ and $n_{y}(t)$ are the ASE noise in the polarizations of $\hat{x}$ and $\hat{y}$, respectively, $A_{L}$ and $\omega_{L O}$ are the amplitude and frequency of the local oscillator, respectively, and $n_{L}(t)$ is the local oscillator optical noise, originated from the relative intensity noise (RIN) of local oscillator laser. The amplifier noise can be expressed as a complex representation:

$$
n_{x}(t)=n_{x 1}(t)+j n_{x 2}(t)
$$

Where $n_{x 1}(t)+$ and $n_{x 2}(t)$ are Gaussian distributed with zero mean and variance $\sigma_{x}^{2}=S_{n_{s}} B_{o} / 2 . S_{n_{s}}$ is the power spectral density of the received spontaneous emission (ASE noise) in each polarization $(\hat{x}$ or $\hat{y})$ and $B_{o}$ is the optical bandwidth of the received optical filter [8]. Notice that $\operatorname{var}\left\{n_{x}(t)\right\}=$ $2 \sigma_{x}^{2}=S_{n_{s}} B_{o}$. In our analysis below we ignore the thermal noise for simplicity. This would give more insight into the problem under consideration. However, as an additive noise, the effect of thermal noise can be added to the signal afterwards. The output current of the balanced detector, neglecting the small effect of beating of ASE noise with LO noise, is given by

$$
\begin{aligned}
i(t)= & 2 \mathcal{R} A_{L} A_{s}(t) \cos \left[\omega_{I F} \mathrm{t}+\phi_{s}(t)\right]+2 \mathcal{R} \mathfrak{N}\left\{\left[A_{L} n_{x}(t)+\right.\right. \\
& \left.\left.A_{s}(\mathrm{t}) e^{j \phi_{s}(t)} n_{L}(t)\right] e^{j \omega_{I F} t}\right\}+i_{s h}
\end{aligned}
$$

Where $\mathcal{R}$ is the photodiode responsively, $\omega_{I F}=\omega_{c}-\omega_{L O}$ is the intermediate frequency, and $i_{s h}$ is the summation of the shot noise currents of the two photodiodes. Generally, the value of signal-LO beating noise is small compared to that of both LO-ASE beating noise and detector shot noise. Furthermore, in the usual case where the LO power is significantly larger than the received power; the dominant noise source is LO-ASE beating. In this case we get an amplifier-noise limited system and the balanced detector current can be simplified as

$$
\begin{aligned}
i(t)= & 2 \mathcal{R} A_{L} A_{s}(t) \cos \left[\omega_{I F} \mathrm{t}+\phi_{s}(t)\right] \\
& +2 \mathcal{R} \mathfrak{N}\left\{A_{L} n_{x}(t) e^{j \omega_{I F} t}\right\} \\
= & 2 \mathcal{R} A_{L} A_{s}(t) \cos \left[\omega_{I F} \mathrm{t}+\phi_{s}(t)\right]+2 \mathcal{R} A_{L} n_{x 1}(t) \cos \omega_{I F} \mathrm{t}- \\
& 2 \mathcal{R} A_{L} n_{x 2}(t) \sin \omega_{I F} \mathrm{t}
\end{aligned}
$$

In a heterodyne receiver, this current would pass to a subcarrier synchronous detector (to remove the effect of the IF frequency), followed by a low-pass filter (LPF) with electrical bandwidth of $B_{e}=1 / 2 T_{s}$. The LPF output can be expressed as

$$
i_{L P F}(t)=\mathcal{R} A_{L} A_{s}(t) \cos \left[\phi_{s}(t)\right]+\mathcal{R} A_{L} n(t)
$$

where, $n(t)$ is a band-limited noise of bandwidth $B_{e}$. Also, it should be noticed that during any time slot within a frame, $A_{S}(t) \in\left\{0, A_{H}\right\}$, for some constant $A_{H}>0$, and $\phi_{S}(t) \in$ $\{0, \pi\}$. That is $A_{s}(t) \cos \left[\phi_{S}(t)\right] \in\left\{-A_{H}, 0, A_{H}\right\}$. Next $i_{L P F}(t)$ 
is passed through an integrator over slot duration. The integrator output over slot $k \in\{1,2, \ldots, M\}$, also called the decision random variable, is

$$
y_{k} \stackrel{\text { def }}{=} \frac{1}{\mathcal{R} A_{L} T_{S}} \int_{(k-1) T_{S}}^{k T_{S}} i_{L P F}(t) d t
$$

$= \begin{cases}A_{H}+n_{k} ; & \text { if a pulse was transmitted, modulated by } 1 \\ n_{k} ; & \text { if a pulse was not transmitted } \\ -A_{H}+n_{k} ; & \text { if a pulse was transmitted, modulated by } 0\end{cases}$

Where $T_{S}$ is the slot duration and

$$
n_{k} \stackrel{\text { def }}{=} \frac{1}{T_{S}} \int_{(k-1) T_{S}}^{k T_{S}} n(t) d t
$$

It is clear that, for any $k \in\{1,2, \ldots, M\}, n_{k}$ is a Gaussian random variable with mean and variance given by

$$
\begin{gathered}
\mu_{n} \stackrel{\text { def }}{=} E\left\{n_{k}\right\}=0 \\
\sigma_{n}^{2} \stackrel{\text { def }}{=} \operatorname{var}\left\{n_{k}\right\}=\frac{1}{2} S_{n_{s}} B_{e}=\frac{S_{n_{s}}}{4 T_{s}},
\end{gathered}
$$

respectively.

In a homodyne receiver, we can get the same decision random variable as given in (10) if we used an integrator $\frac{1}{2 \mathcal{R} A_{L} T_{S}} \int_{(k-1) T_{S}}^{k T_{S}}($.$) rather. For either a heterodyne or homodyne$ receiver, the output of slot integrator $y_{k}$ is one of three Gaussian random variables, which are $A_{H}+n_{k}, n_{k}$, or $-A_{H}+$ $n_{k}$, associated with the transmission of an optical pulse modulated by one, transmission of no optical pulse, or transmission of an optical pulse modulated by zero, respectively. The probability distributions of $y_{k}$ for these three cases are given by

$$
\begin{gathered}
p_{1}\left(y_{k}\right) \stackrel{\text { def }}{=} p\left(y_{k} \mid 1\right)=\frac{1}{\sigma_{n} \sqrt{2 \pi}} e^{-\left(y_{k}-A_{H}\right)^{2} / 2 \sigma_{n}^{2}} \\
p_{0}\left(y_{k}\right) \stackrel{\text { def }}{=} p\left(y_{k} \mid 0\right)=\frac{1}{\sigma_{n} \sqrt{2 \pi}} e^{-y_{k}{ }^{2} / 2 \sigma_{n}^{2}} \\
p_{-1}\left(y_{k}\right) \stackrel{\text { def }}{=} p\left(y_{k} \mid-1\right)=\frac{1}{\sigma_{n} \sqrt{2 \pi}} e^{-\left(y_{k}+A_{H}\right)^{2} / 2 \sigma_{n}^{2}}
\end{gathered}
$$

respectively. The receiver gets the $n_{H}$ slots with the highest energy in the received hybrid frame by squaring the integrator output. The resultant random variable $z_{k}=y_{k}^{2}$ takes one of two cases: $\left(A_{H} \pm n_{k}\right)^{2}$ or $n_{k}^{2}$, depending on the transmission of an optical pulse or no optical pulse, respectively. The probability distribution of $z_{k}$ in the case of transmitting an optical pulse is a non-central chi-square $\chi^{2}$ with one degree of freedom, given by

$$
\begin{aligned}
p_{1}\left(z_{k}\right) \stackrel{\text { def }}{=} p\left(z_{k} \mid 1\right)= & \frac{1}{2 \sigma_{n}^{2}} e^{-\left(z_{k}+A_{H}^{2}\right) / 2 \sigma_{n}^{2}}\left(\frac{A_{H}^{2}}{z_{k}}\right)^{\frac{1}{4}} \times \\
& \frac{I_{-1}}{2}\left(\frac{A_{H}}{\sigma_{n}^{2}} \sqrt{z_{k}}\right), \quad z_{k}>0
\end{aligned}
$$

where $I_{\alpha}(v)$ is the $\alpha$ th-order modified Bessel function of the first kind, while, the probability distribution of $z_{k}$ in the case of no optical pulse transmission, is a central chi-square with one degree of freedom (also called gamma distribution), given by

$$
p_{0}\left(z_{k}\right) \stackrel{\text { def }}{=} p\left(z_{k} \mid 0\right)=\frac{1}{\sigma_{n} \sqrt{2 \pi z_{k}}} e^{-z_{k} / 2 \sigma_{n}^{2}}, \quad z_{k}>0
$$

Clearly, the receiver selects the $n_{H}$ slots with the highest values of $z_{k}$ (highest energies) and decodes them as the transmitted signal pulses. The probability of error in such decoding is the same as the symbol-error rate of the ordinary MPPM scheme, which is given by [11], with slight modifications. We proceed as follows. The probability of correct symbol detection equals to the probability that $n_{H}$ signal slot energies are all greater than the largest energy of $M-n_{H}$ non-signal slot. Let $Z$ be the largest non-signal slot energy, and then the probability of errors in such symbol decoding is given by

$$
\begin{aligned}
\operatorname{SER}_{\mathrm{MPPM}}= & 1-\int_{0}^{\infty}\left(M-n_{H}\right) P_{0}(Z)^{M-n_{H}-1} \times p_{0}(Z)[1- \\
& \left.P_{1}(Z)\right]^{n_{H}} d Z,
\end{aligned}
$$

where, $p_{0}(Z)$ and $p_{0}(Z)$ are the probability density functions (pdfs) of $Z$ in signal and non-signal slots, and are given by (14) and (15), respectively. Also, $P_{1}(Z)$ and $P_{1}(Z)$ denote its cumulative distributions for both signal and non-signal slots, respectively. Clearly, the equation considers all probabilities for $M-n_{H}$ non-signal slots to have energy less than the $n_{H}$ signal slots.

After getting the positions of the $n_{H}$ signal slots in the received hybrid frame, the receiver proceeds to decode these BPSK modulated signal slots to find the remaining $n_{H}$ bits of the transmitted data block. Clearly, the decoding decision will be based on the values of slot integrator for the predetermined signal slots. The integrator output of these slots takes one of two values which are $A_{H}+n_{k}$ or $-A_{H}+n_{k}$, depending on the transmission of data bit one or zero on the optical pulse, respectively. With the optimal threshold set at zero, the error probability in decoding these BPSK symbols (or bits) is the same as the bit error rate of ordinary BPSK [12]:

$$
\begin{gathered}
\operatorname{BER}_{\mathrm{BPSK}}=\frac{1}{2} \int_{-\infty}^{0} p\left(y_{k} \mid 1\right) d y+\frac{1}{2} \int_{0}^{\infty} p\left(y_{k} \mid-1\right) d \\
=\frac{1}{2} \operatorname{erfc}\left(\frac{A_{H}}{\sigma_{n} \sqrt{2}}\right)
\end{gathered}
$$

Substituting (16) and (17) into (4), we obtain the exact $S_{E R}$ for the proposed hybrid BPSK-modified MPPM scheme.

For the purpose of performance comparison, we recall the SER expressions for both coherent and direct detection receivers of MPPM scheme. Clearly, the SER for each receiver is still given by equation (16), but with different 
distributions, integration limits, and different number of optical pulses per frame $n_{M}$. For direct detection of MPPM frames, the distributions are given in [10] and [13], while for coherent detection of MPPM frames, the distributions are given by

$$
\begin{gathered}
p_{1}\left(z_{k}\right)=\frac{1}{\sigma_{n} \sqrt{2 \pi}} e^{-\left(z_{k}-A_{M}\right)^{2} / 2 \sigma_{n}^{2}} \\
p_{0}\left(z_{k}\right)=\frac{1}{\sigma_{n} \sqrt{2 \pi}} e^{-z_{k}^{2} / 2 \sigma_{n}^{2}}
\end{gathered}
$$

where, $A_{M}$ is the amplitude of demodulated signal in ordinary MPPM schemes (for both coherent and direct detection).

\section{NumERICAL RESULTS}

In this section we compare the performance achieved by the proposed hybrid BPSK-modified MPPM scheme with that achieved by ordinary MPPM scheme. The comparison is carried out in terms of symbol-error rate. Although, the comparison is evaluated for optical fiber channels, it can be extended to other optical channels by including the appropriate optical transmission model. For fair performance comparison, we assume the usage of same frame size and same transmission rate for both schemes. Specifically, the evaluations are performed at two different frame sizes, namely, $M=12$ and $M=24$ slots. For the case of $M=12$, two comparisons are carried out. Firstly, 3-pulse 12-PPM is compared to hybrid 2-BPSK 12-PPM and secondly, 6-pulse 12-PPM is compared to hybrid 3-BPSK 12-PPM. For these hybrid schemes, the numbers of transmitted bits per frame are 8.04 bits and 10.78 bits, respectively, which are slightly larger than that of the ordinary MPPM schemes (7.78 bits and 9.85 bits, respectively). Clearly, these selections achieve nearly the same transmission rates for both hybrid and ordinary schemes with larger rates for the hybrid ones. For the case $M=24$, two comparisons are also performed. Particularly, we compare 6-pulse 24-PPM scheme to hybrid 4-BPSK 24-PPM scheme and compare 12-pulse 24-PPM scheme to hybrid 6-BPSK 24PPM scheme. Again, in these comparisons, the hybrid schemes achieve higher transmission rates than that of ordinary MPPM schemes, which represents an extra advantage in favor of selected hybrid schemes. In terms of bandwidth utilization efficiency, the aforementioned selections result in near same bandwidth utilization efficiency for both schemes. However, the hybrid schemes can even achieve higher bandwidth utilization efficiencies than that of ordinary MPPM schemes by increasing the number of transmitted optical pulses in the hybrid frames above the selected values.

The bit encoding for hybrid BPSK-modified MPPM can be realized using nearly the same encoding techniques of ordinary MPPM. The only difference is to encode extra $n_{H}$ bits in each hybrid frame. However, there is no known efficient method of encoding MPPM symbols [14]. The simplest, but inefficient, encoding scheme is to encode each symbol by the integer number of bits resulting from taking the logarithm of the number of available symbols in the system. As an example of such encoding, consider the case of the 3-pulse 12-PPM scheme, each frame is encoded only by 7 bits and the remaining fraction of bits $(0.7814$ bits $)$ is discarded. Clearly, in this case not all available symbols are used for transmission and there is an optimal bit-symbol mapping that results in the lowest BER. Another simple and more efficient encoding scheme is to create compound symbols by cascading enough MPPM symbols to ensure that the total number just exceeds a power of 2 and then encode suitably larger blocks of information bits into this compound signal set [15]. The bit encoding and decoding for both the hybrid and ordinary MPPM along with the transmitter and receiver complexity are not in the scope of this paper. However, they represent important design issues that could be considered in other research works

Generally, coherent detection results in a better receiver sensitivity than direct detection, but it requires more complex receiver structure. In the carried evaluations below, we compare the performance achieved by the proposed coherent detection hybrid BPSK-modified MPPM scheme to that achieved by both coherent detection and direct detection MPPM schemes. Also, in all these cases, the system noise is assumed to be dominated by optical amplifier ASE noise. Furthermore, our comparisons are performed under the average power constraint which is imposed in many optical communications systems. For optical fiber based systems, it rises in the case of long haul transmission where optical repeaters with limited power are used and also in the case of DWDM transmission where thousands of optical carriers are launched together in a single core and the total average power launched to the fiber must not exceed a maximum value. For non-fiber systems, the average power constraint is imposed when the transmitter has limited power resources. This occurs in free-space optical systems working with solar energy and space optical communications between spacecrafts. Clearly, the modulation scheme that achieves higher performance at the same average power level is highly desirable in these cases.

Here, the average power for hybrid BPSK-modified MPPM and ordinary MPPM schemes can be expressed as $P_{a v H}=$ $A_{H}^{2} \cdot n_{H} / M$ and $P_{a v M}=A_{M}^{2} \cdot n_{M} / M$, respectively. Clearly, under the average power constraint, we have $P_{a v H}=P_{a v M}$ and

$$
A_{H}^{2}=A_{M}^{2} \frac{n_{M}}{n_{H}}
$$

It should be noticed that with same transmission rates for both hybrid and ordinary schemes, the average power constraint implies transmission of same energy per bit. In this case, the average received signal-to-noise ratio for both schemes is given by

$$
\mathrm{SNR}_{a v}=\frac{P_{a v}}{\sigma_{n}^{2}}=\frac{A_{H}^{2} \cdot n_{H}}{M \sigma_{n}^{2}}=\frac{A_{M}^{2} \cdot n_{M}}{M \sigma_{n}^{2}}
$$

The symbol-error rates achieved by the proposed hybrid scheme and the ordinary MPPM schemes (both coherent and direct detection) are plotted versus the average signal-to-noise ratio $\left(\mathrm{SNR}_{a v}\right)$ in Figs. 6 and 7 for the cases $M=12$ and $M=$ 24 , respectively. From these figures it is clear that at all levels of $\mathrm{SNR}_{a v}$, the hybrid schemes outperform the ordinary MPPM 


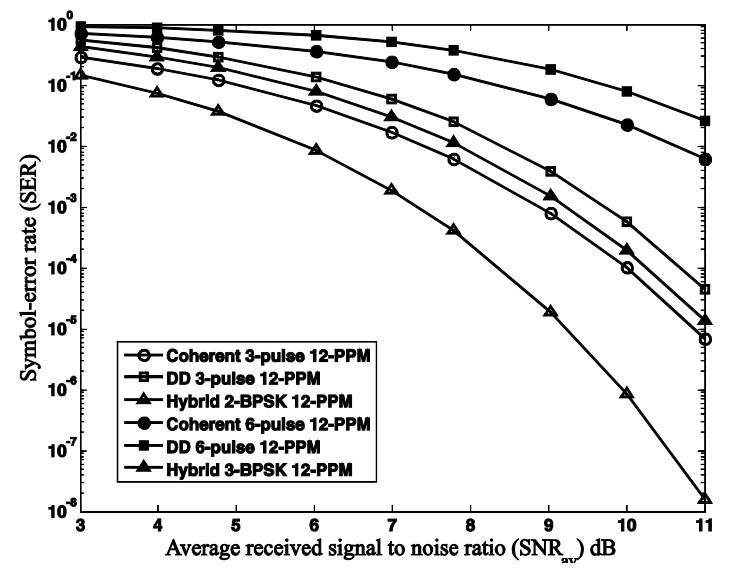

Fig. 6. Symbol-error rate versus average received signal-tonoise ratio at $M=12$.

schemes for both coherent and direct detection. Numerically, as indicated in Fig. 6, at $\mathrm{SNR}_{a v}=10 \mathrm{~dB}$, the hybrid 2-BPSK 12-PPM scheme achieves more than two order of magnitude less SER than those of the coherent detection and direct detection 3-pulse 12-PPM schemes. Also as expected, the coherent detection MPPM schemes have better performance than that of the direct detection ones. Furthermore, the performance gap between the hybrid schemes and ordinary ones is significantly increased by raising the level of $\mathrm{SNR}_{a v}$. Specifically, for coherent 3-pulse 12-PPM and hybrid 2-BPSK 12-PPM schemes, raising the $\mathrm{SNR}_{a v}$ level from $6 \mathrm{~dB}$ to $8 \mathrm{~dB}$ nearly doubles the performance gap between both schemes. This may be interpreted as follows. With same average receiving power, raising the $\mathrm{SNR}_{a v}$ level results in noticeable increasing in the received pulse power for the hybrid scheme over the ordinary ones which in turn leads to better signal detection and larger performance gap. Also as expected, increasing the number of optical pulses per frame degrades the achieved performance. Obviously, this comes from the fact that with the same $\mathrm{SNR}_{a v}$ level increasing number of optical pulses per frame reduces the received slot power, which in turn decreases the capability of correct decoding.

Moreover, with the same number of optical pulses per frame, increasing the frame size would result in better performance. It is clear from Figs. 6 and 7 that, at the same levels of $\mathrm{SNR}_{a v}$, the performance of the hybrid 4-BPSK 24PPM scheme(worse than that of the hybrid 3-BPSK 24-PPM scheme) is always better than that of the hybrid 3-BPSK 12PPM scheme. This is because with same $\mathrm{SNR}_{a v}$ level and same number of optical pulses per frame, increasing the frame size from 12 to 24 slots results in doubling the pulse power, which in turn increases the probability of correct decoding for the transmitted frame. The analysis of the previously achieved SERs could be further explained using Figs. 8 and 9. These figures indicate the comparison between hybrid and ordinary schemes in terms of the peak received power. Fig. 8. indicates the comparison for the case of $M=12$, while Fig. 9. indicates it for the case of $M=24$. Obviously, at the same transmission rates for both hybrid and ordinary MPPM schemes, the number

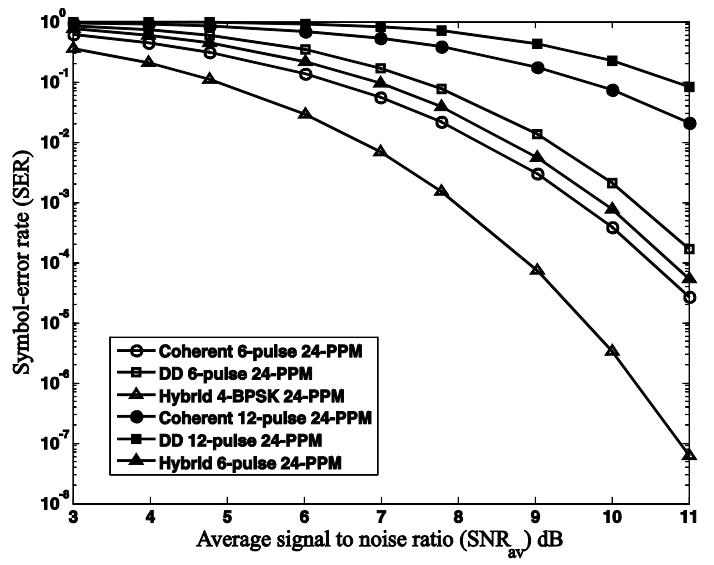

Fig.7. Symbol-error rate versus average received signal-tonoise ratio at $M=24$.

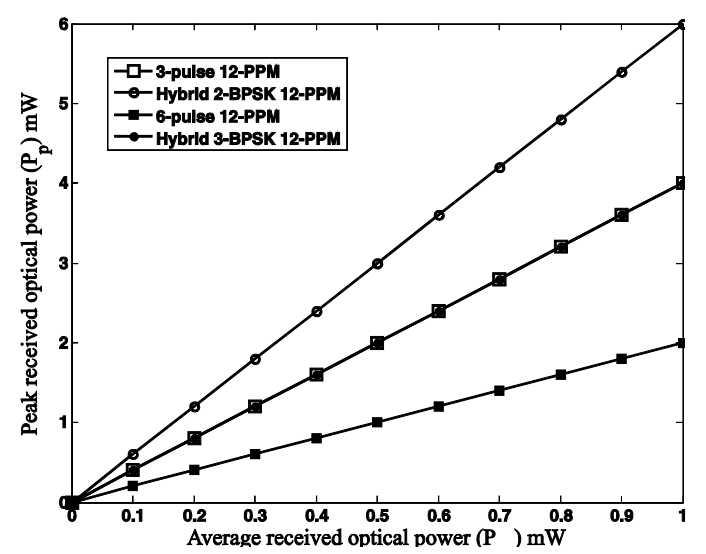

Fig. 8. Peak received optical power versus average received power at $M=12$

of signal slots per frame in the hybrid frames is less than that of the ordinary MPPM schemes. Thus, when receiving same average power per frame, a noticeable increasing in the pulse power (peak power) is achieved for hybrid schemes. Particularly, for hybrid 2-BPSK 12-PPM scheme, the peak received power is increased linearly versus average received power with a rate equals to one and half the rate of 3-pulse 12PPM scheme. Moreover, increasing the number of optical pulses in hybrid frames raises the rate of increasing in the peak power. Clearly, for hybrid 3-BPSK 12-PPM scheme, the rate of increasing in the peak power is twice the rate of 6-pulse 12PPM scheme. Thus, the enhancement in the performance achieved by the hybrid schemes comes with the price of increasing peak power levels. Obviously, these high levels of received peak power could raise the levels of both nonlinear distortion and cross phase noise which are not desirable for high speed optical fiber communications. Therefore, the increase in the peak power for hybrid scheme may limit its performance to values lower than that of the ordinary schemes when the same maximum peak power level is supported. 


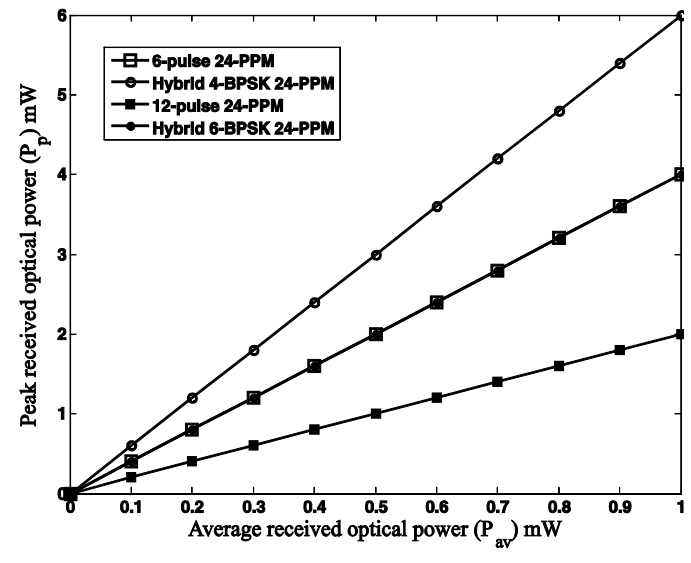

Fig. 9. Peak received optical power versus average received power at $M=24$.

\section{CONCLUSION}

A new hybrid modulation scheme, hybrid BPSK-modified MPPM, has been proposed for optical fiber communications. The proposed scheme achieves much higher bandwidth utilization efficiency than that of the ordinary MPPM scheme. Both the transmitter and receiver structures along with the optimal decoding algorithm have been presented and discussed for optical fiber channel systems. The performance measure of the proposed scheme in terms of exact symbolerror rate has been derived taking into account the effect of optical fiber noise. Intensive numerical evaluations have been performed to compare the performance achieved by the proposed hybrid scheme with that of both coherent and direct detection MPPM schemes. The numerical evaluations have been carried out under the average power constraint at nearly the same transmission rates for hybrid and ordinary schemes. Our results indicate the superior performance of the proposed hybrid scheme under average power constraints over both coherent and direct detection MPPM schemes.

\section{REFERENCES}

[1] H. Sugiyama and K. Nosu, "MPPM: A method for improving the band- utilization efficiency in optical PPM," Journal of Lightwave Technology, vol. 7, no. 3, pp. 465-471, March 1989.

[2] J. Singh and V. K. Jain, "Performance analysis of BPPM and M-ary PPM optical communication systems in atmospheric turbulence," IETE Tech. Rev., vol. 25, no. 4, pp. 146-153, July-Aug. 2008

[3] G. E. Atkin and K. S. Fung, "Performance analysis of coded optical PPM system using direct and coherent detection," IEE Proceedings I:Communications, Speech and Vision, vol. 137, no. 4, pp. 226-232, Aug. 1990.

[4] M. M. Fern'andez and V. A. Vilnrotter. "Performance analysis and preliminary experimental verification of a coherent optical receiver for PPM signals in the presence of atmospheric turbulence," Tech. Report, Jet Propulsion Laboratory, National Aeronautics and Space Administration, March 2004.

[5] V. A. Vilnrotter and C. Lau, "Quantum detection and channel capacity for communications applications," Proc. SPIE 4635, pp. 103-115 (2002).

[6] M. Faridzadeh, A. Gholami, Z. Ghassemlooy and S. Rajbhandari, "Hybrid PPM-BPSK subcarrier intensity modulation for free space optical communications," 16th European Conference on Networks and Optical Communications (NOC 2011), pp. 36-39, July 20-22, 2011.

[7] X. Liu, T. H. Wood, R. W. Tkach, and S. Chandrasekhar, "Demonstration of record sensitivity in an optically pre-amplified receiver by combining PDM-QPSK and 16-PPM with pilot-assisted digital coherent detection," OFC 2011, PDPB1 (2011).

[8] K.-P. Ho, "Phase-modulated optical communication systems," New York, Springer, 2005.

[9] H. Park, "Performance bound on multiple-pulse position modulation," Optical Review, vol. 10, no. 3, pp. 131-132, May 2003.

[10] X. Liu, S. Chandrasekhar, and A. Leven, "Self-coherent optical transport systems," in Optical Fiber Telecommunications V B, Systems and Networks, I. P. Kaminow, T. Li, and A. E. Willner, Eds. Burlington, MA: Academic, 2008, ch. 4.

[11] J. Hamkins and B. Moision, "Multipulse PPM on memoryless channels,"in International Symposium on Information Theory (ISIT), Chicago, Illinois, p. 336, June 2004.

[12] .J. G. Proakis, Digital communications, 4th ed. Boston, MA: Mc-GrawHill, 2001.

[13] X. Liu, S. Chandrasekhar, T. H. Wood, R. W. Tkach, P. J. Winzer, E.C. Burrows, and A. R. Chraplyvy, "M-ary pulse-position modulation andfrequency-shift keying with additional polarization/phase modulation for high-sensitivity optical transmission," in Opt. Express 19, B868B881 (2011).

[14] S. Liu and F.R. Kschischang, "Coding for MPPM-like systems," 2010 25th Biennial Symposium on Communications (QBSC 2010), 12-14 May 2010, Kingston, Ontario, Canada.

[15] M. Simon and V. Vilnrotter, "Performance analysis and tradeoff for dual-pulse PPM on optical communications channels with direct detection,"IEEE Trans. Commun., vol. 52, no. 11, pp. 1969-1979, November 2004. 